This item was submitted to Loughborough's Research Repository by the author.

Items in Figshare are protected by copyright, with all rights reserved, unless otherwise indicated.

\title{
Talent development environment and achievement goal adoption among Korean and Singaporean athletes: Does perceived competence matter?
}

PLEASE CITE THE PUBLISHED VERSION

http://dx.doi.org/10.1177/1747954116654779

\section{PUBLISHER}

(c) The Authors. Published by Sage.

\section{VERSION}

AM (Accepted Manuscript)

\section{PUBLISHER STATEMENT}

This work is made available according to the conditions of the Creative Commons Attribution-NonCommercialNoDerivatives 4.0 International (CC BY-NC-ND 4.0) licence. Full details of this licence are available at: https://creativecommons.org/licenses/by-nc-nd/4.0/

\section{LICENCE}

CC BY-NC-ND 4.0

\section{REPOSITORY RECORD}

Wang, C.K. John, Do Young Pyun, Chunxiao Li, and M.S. Lee. 2019. "Talent Development Environment and Achievement Goal Adoption Among Korean and Singaporean Athletes: Does Perceived Competence Matter?". figshare. https://hdl.handle.net/2134/20863. 
Running head: TALENT DEVELOPMENT ENVIRONMENT AND ACHIEVEMENT GOALS

Talent Development Environment and Achievement Goal Adoption among Korean and Singaporean Athletes: Does Perceived Competence Matter? 
ABSTRACT

The objectives of this study were twofold. The first was to compare differences in perceptions of the talent development environment, achievement goals, and perceived competence, in terms of an individual characteristic (i.e., gender), a cultural characteristic (i.e., country), and their interactions (i.e., gender by country). The second was to examine the moderating effects of perceived competence on the relationships between the talent development environment and achievement goals. Data were collected from 363 athletes in Singapore and 349 athletes in Korea. A series of MANOVAs and path analyses were employed for testing of the main hypotheses. First, in terms of the talent development environment, male athletes scored higher in long term development focus, communication, support network, and long term development fundamentals. Second, in terms of achievement goals and perceived competence, male athletes scored higher in competence and performance-approach goal, but female athletes scored higher in masteryavoidance goal. While Singaporean athletes scored higher in perceived competence, masteryapproach and mastery-avoidance, Korean athletes scored higher in performance-avoidance. Lastly, the path analysis provided empirical evidence supporting the moderation effects of perceived competence on the relationships between the talent development environment and achievement goal adoption.

Key words: talent development environment, achievement goal, perceived competence 


\section{INTRODUCTION}

To achieve international sporting success, many countries have adopted and developed systematic talent identification and development (TID) programs in the quest to maximize their athletes' potential [1]. Countries such as United States, United Kingdom, Australia, South Korea, Japan, China, and recently Singapore have invested a large amount of resources into TID programs [2]. For example, the Korea Foundation for the Next Generation Sports Talent, which was founded in 2007, develops sport talents for international sporting excellence. UK Sport spends about $£ 100$ million of public funds every year to achieve high performance sport. Alongside the huge investments, athletes are promised attractive monetary rewards. For example, a Singapore Olympic gold medalist can receive S\$1 million dollars (around US\$800,000), and Italy pays its gold medalist up to about US\$182,000.

Past literature showed clear evidence that both talent identification (nature) and talent development (nurture) are critical factors for achieving peak performance [2]. The recent trend in TID research is moving toward talent development rather than talent identification partially because research has demonstrated that spending time on testing or searching sport talents may not be productive $[1,3]$. Rather, talent development provides a suitable learning environment in which the athletes are given the opportunity to realize and nurture their potential.

\section{EFFECTIVE TALENT DEVELOPMENT ENVIRONMENT}

For investigations of effective talent development, several models have been proposed to better understand the process of achieving excellent performance [4]. For example, the theory of deliberate practice hypothesizes that at least 10,000 hours of practice are necessary to attain expertise in one specific domain [5], which emphasizes a long-term progression for sport talent development and the significance on understanding the process. Another example is the stage 
model proposed by Bloom [6]. This model addresses that there are three stages of talent development (i.e., initiation, development, and perfection). The movement from one stage to another stage is determined when certain tasks required in each stage are completed, attitudes/networks are developed, and relevant skills are learned, rather than the chronological age. Bearing this in mind, the progression of talent development could vary among different athletes due to individual differences.

To account for individual differences, several environmental factors that could help to shape the athletes' development processes for expert performance were conceptualized [3]. Martindale et al. [7] recently developed a scale entitled the Talent Development Environment Questionnaire assessing seven key factors of the effective talent development environment based on literature review, content analysis, and interviews with coaches and athletes. The first factor is long term development focus which refers to the extent to which the training program is developmental in nature, where the focus is on long term success. This factor also represents attitudes, psychological skills, and understanding required for long term success (e.g., responsibility, dedication, mental skills, and learning through mistakes). The second factor is quality preparation. It refers to the availability of clear guidance and opportunities for quality practice through training, recovery, and competition experiences. The third and fourth factors are communication and understanding the athletes. While communication refers to the interaction between the coach and athlete in both formal and informal settings, understanding the athletes is defined as the extent to which the coach understands the athlete in depth at a holistic level, and has developed a strong professional relationship with them. The fifth factor is support network, which is related to a coherent, approachable and variety of support network to help and support the athletes' development in all areas. The sixth factor is challenging and supportive 
environment which refers to the extent to which athletes are challenged appropriately in training and competitions to facilitate their development to the highest level. The final factor is long term development fundamentals, which reflects to the extent to which key features of the foundations for further development are considered, such as ongoing opportunities, avoidance of early specialization, parental support, and athletes' decision making. In the recent study, Wang and his colleagues proposed the six-dimensional TDEQ scale excluding challenging and supportive environment due to its poor reliability [8]. The rationales behind the exclusion are its internal consistency was unsatisfactory, and it was deemed that this factor was conceptually overlapped with quality preparation and support network [8].

While different environmental factors are expected to influence elite athletes at the micro (e.g., gender) or macro (e.g., culture) level, previous research has not examined the possible effects of individual and cultural characteristics on perceptions of the talent development environment. Therefore, the first objective of this study was to see if there was any difference in the six talent development factors in term of gender, country, and their interactions.

\section{ACHIEVEMENT GOALS}

The talent development environment is a significant indicator of athletes' goal pursuits as well as achievement goals [8]. According to the traditional achievement goal theory, there are at least two different ways to define competence or success, and they are embedded in the goals that one seeks to achieve in a specific athletic setting [9]. The first type of goal, labeled as 'mastery' or 'task' goal, represents self-referenced mastery or indicates how to perform the task and is labeled as 'mastery' or 'task' goal [10]. The second type of goal emphasizes normative comparison of ability or performance relative to others and is therefore labeled as 'ego' or 'performance' goal [10]. In a recent re-conceptualization of achievement goals, Elliot and his 
colleagues [11-13] proposed that achievement goals could be formulated as a $2 \times 2$ framework by adding an approach-avoidance dimensions to the mastery-performance dimension: masteryapproach (task-based or intrapersonal competence), mastery-avoidance (task-based or intrapersonal incompetence), performance-approach (normative competence), and performanceavoidance (normative incompetence). Previous research has established that both masteryapproach and performance-approach goals are proposed to contribute to positive effects and consequences [12]. Mastery-avoidance and performance-avoidance goals produce less adaptive motivational patterns such as disorganization, worry, and emotionality [13-15].

Researchers in achievement goal theory hypothesized that the processes initiated by the adoption of achievement goals differed on gender, but past research findings were mixed [16]. In a systematic review of achievement goal research, Biddle and his colleagues found that females showed higher level of mastery goals than their counterparts, and males reported higher performance goals in sport and physical education contexts [17]. While there has been no empirical research to test gender differences in the avoidance dimension of achievement goals, it was assumed that given the process of socialization, females may display less achievement motivation compared to males [18].

Additionally, given the achievement goal framework proposed by Elliot $[11,12]$, perceived competence is differentiated in two ways. First, competence is defined by an individual's standard used to evaluate his or her ability in a specific area. The standard is either the task itself related to one's own past performance (mastery) or the performance of others (performance). Second, competence can be differentiated by its valence. That is, whether the focus is on a positive possibility (approach) or a negative possibility (avoidance). Thus, competence is viewed as an antecedent of achievement goals. Wang et al. [8] showed that high 
competency athletes are more likely to adopt mastery-approach and performance-approach goals, compared to low competency athletes. Therefore, it is worthwhile to examine moderating effects of perceived competence on the relationships between the talent development environment and achievement goals adaption. This is the second purpose of the study.

In summary, the purpose of the current study was twofold. First, the authors examined the effects of gender and country on the talent development environment, achievement goals, and perceived competence. Korea and Singapore are two countries with different cultures in Asia. No previous studies have examined the similarities and differences between the two countries. There is a call to consider the intersection of gender and cultural contexts in which beliefs develop and change over time when studying competence and achievement motivation [16]. For instance, female athletes are stereotyped to be more mastery goals oriented while male athletes are assumed to be more performance goals oriented. Similarly, based on the cultural norm, Singaporean athletes are likely to show higher mastery goals but Korean athletes may tend to exhibit higher performance goals. Thus, gender and culture may produce a "double-dose of stereotype threat" (16, p. 388), saying that Singaporean female athletes could be double stereotyped to form the highest level of mastery goals. It would be also worthwhile to see if gender and culture could create accumulated influences on the talent development environment and achievement goals.

Second, since the achievement goal theory proposed that levels of perceived competence determine the adoption of achievement goals, it is important to examine moderating effects of competence on the relationships between the talent development environment and achievement goals. The following research questions were formulated in the current study: 
1. Are there gender and country differences in the perceptions of the talent development environment and adoption of achievement goals?

2. What is the role of the competence in the subject's perceptions of the talent development environment and adoption of achievement goals?

3. Does competence moderate the relationships between the talent development environment factors and adoption of achievement goals?

\section{METHOD}

\section{PARTICIPANTS}

The data were collected from young elite athletes attending sports schools in Singapore and Korea. Athletes from Singapore $(n=363)$ and Korea $(n=349)$ took part in this study. Among them, there were 414 male athletes and 292 female athletes. Six athletes did not indicate their gender. Their ages ranged from 12 to 16 years old $(M=14.82, S D=0.9)$. The subjects were participating in various sports (e.g., weightlifting, taekwondo, judo, and volleyball) with a mean of 5.08 years $(S D=2.00)$ of training experience.

\section{DATA COLLECTION PROCEDURES}

Permission to conduct the survey was secured from head-teachers of two sports schools each in Singapore and Korea. The both schools have run the talent development programs. Studentathletes were informed to participate in the survey voluntarily, free to withdraw at any time, and were assured that their responses would be kept confidential. All students were required to submit written informed consent sought from their parents or guardians. The survey took approximately 10 minutes to complete. The questionnaires used for the Korean subjects were 
translated and back-translated by two bilingual Korean scholars in the areas of sport psychology and coaching, based on the Brislin's [19] guidelines.

\section{MEASURES}

\section{Talent development environment questionnaire (TDEQ).}

The six-dimensional TDEQ with 36 items, proposed by Wang et al. [8], was utilized to measure the talent development environmental factors. The scale included six factors: long-term development focus (five items), lack of quality preparation (five items), communication (seven items), lack of understanding the athlete (four items), support network (eight items), and longterm development fundamentals (seven items). Responses were made on a 6-point Likert scale, anchored strongly disagree (1) and strongly agree (6).

The $2 \times 2$ achievement goals in physical education questionnaire (AGPEQ).

The AGPEQ was adapted to measure the four achievement goals in elite sports [20]. There were 12 items in the scale with three items each to measure the four achievement goals: masteryapproach (e.g., 'I want to perform as well as it is possible for me to perform'), masteryavoidance (e.g., 'I am often concerned that I may not perform as well as I can perform'), performance-approach (e.g., 'It is important for me to do well compared to others'), and performance-avoidance (e.g., 'My goal is to avoid performing worse than everyone else'). Responses were made on a 7-point Likert scale ( $1=$ strongly disagree, $7=$ strongly agree $)$.

\section{Perceived competence.}

The competence subscale with five items in the Basic Psychological Needs Scale [21] was used to assess the satisfaction of need of competence in the talent development environment. A 7point Likert scale $(1=$ not true at all, $7=$ very true $)$ was used for making responses. The alpha coefficient was .78 for competence. 


\section{DATA ANALYSIS}

Confirmatory factor analysis (CFA) was conducted to test the factorial validity of the TDEQ and AGPEQ using EQS for Windows 6.2 [22]. The internal consistency coefficients of the measures were also calculated for the purpose of reliability test. Descriptive statistics and the Pearson product-moment correlations of the main variables were tabulated. A series of two-way Multivariate analysis of variance (MANOVA) were conducted to examine differences between genders, two countries, and their interaction effects. The first MANOVA examined differences in six talent development factors, and the second MANOVAs tested the four achievement goals and perceived competence.

To test for the moderating role of perceived competence on the proposed relationships between the six talent development environment factors and achievement goals, the samples were split into higher and lower competency groups. As the sample size was moderate, the samples were categorized with the 40th and 60th percentiles. A higher competency group had competence higher than $5.33(n=250)$, and a lower competency group had competence lower than $4.68(n=278)$. Thereafter, a paired t-test was conducted to examine if a mean difference between the two competency groups was statistically significant. A MANOVA was conducted to examine if the mean differences between the two competency groups was significant in the various constructs. A chi-square test was also conducted to examine the gender and country effects among the two competency groups. As the final analysis, after examining zero-order correlations among the constructs in both high and low competency groups separately, a series of path analyses were carried out to examine the impacts of the talent development environment on each achievement goals in each group to test the moderation effects of perceived competence. 


\section{RESULTS}

CONFIRMATORY FACTOR ANALYSIS

Prior to conducting the main analyses, two CFAs were conducted independently to examine the adequacy of model fit and reliability of the TDEQ and the AGPEQ. For the TDEQ, the CFA of the first order six-factor structure showed adequate fit to the proposed model $\left(\right.$ Scaled $\chi^{2}=$ $976.15, d f=572, \mathrm{CFI}=.94, \mathrm{IFI}=.94, \mathrm{NNFI}=.94, \mathrm{RMSEA}=.03,90 \% \mathrm{CI}$ of RMSEA $=.03$ to .04 ). The internal reliability for the other six subscales ranged from .73 to .86 (long term development focus, $\alpha=.81$; quality preparation, $\alpha=.73$, communication, $\alpha=.86$, understanding the athletes, $\alpha=.80$. support network, $\alpha=.86$, and long term development fundamentals, $\alpha=$ .82). For the AGPEQ, the CFA showed acceptable fit indices (Scaled $\chi^{2}=172.95, d f=48, \mathrm{CFI}=$ $.96, \mathrm{IFI}=.96, \mathrm{NNFI}=.95, \mathrm{RMSEA}=.06,90 \% \mathrm{CI}$ of RMSEA $=.05$ to .07$)$. The internal reliability coefficients of the four achievement goals were satisfactorily; mastery-approach ( $\alpha=$ $.81)$, mastery avoidance $(\alpha=.86)$, performance approach $(\alpha=.83)$, and performance avoidance $(\alpha=.80)$. No modifications were made to the measurement models during the CFAs.

\section{MULTIVARIATE ANALYSIS OF VARIANCE}

Table 1 shows the descriptive statistics of the main variables calculated from the overall samples as well as by gender and by country. In general, athletes from both countries reported moderately high scores on the TDEQ subscales ( $M=4.52$ to 4.71 , out of 6.00 ), except for lack of quality preparation $(M=3.05)$ and lack of understanding the athletes $(M=3.45)$. They also endorsed high mastery-approach, mastery-avoidance, performance-approach and perceived high competence, ranging from 5.07 to 5.72 out of 7.00 ).

In the first MANOVA, the talent development environment factors were considered as dependent variables. The results showed that there were significant gender differences (Wilk's $\Lambda$ 
$\left.=.96, F[6,697]=4.32, p<.01, \eta^{2}=.04\right)$ and country differences (Wilk's $\Lambda=.62, F[6,697]=$ $71.42, p<.01, \eta^{2}=.38$ ) in the talent development environment factors. There were no interaction effects. In terms of gender, male athletes scored higher in long term development focus $\left(F[1,702]=10.24, p<.01, \eta^{2}=.01\right)$, communication $\left(F[1,702]=22.68, p<.01, \eta^{2}=.03\right.$, support network $\left(F[1,702]=14.80, p<.01, \eta^{2}=.02\right)$, and long term development fundamentals $\left(F[1,702]=12.45, p<.01, \eta^{2}=.02\right)$, compared to the female athletes. When Singaporean athletes and Korean athletes were compared, Singaporean athletes scored higher in lack of quality training $\left(F[1,702]=341.87, p<.01, \eta^{2}=.33\right)$, and lack of understanding the athletes $\left(F[1,702]=318.89, p<.01, \eta^{2}=.31\right)$, compared to the Korean athletes.

The results of the second MANOVA showed that there were significant gender differences (Wilk's $\Lambda=.95, F[5,690]=7.57, p<.01, \eta^{2}=.05$ ) and country differences (Wilk's $\left.\Lambda=.78, F[5,690]=39.91, p<.01, \eta^{2}=.22\right)$ in the four achievement goals and perceived competence. There were no interaction effects. In terms of gender, male athletes scored higher in perceived competence $\left(F[1,694]=8.48, p<.01, \eta^{2}=.01\right)$, and performance-approach goal $(F[1$, $694]=11.34, p<.01, \eta^{2}=.02$ ), compared to the female athletes. On the other hand, the female athletes scored higher in the mastery-avoidance goal $\left(F[1,694]=6.44, p<.05, \eta^{2}=.01\right)$, compared to their male counterparts. When Singaporean athletes and Korean athletes were compared, Singaporean athletes scored higher in perceived competence $(F[1,694]=47.04, p<$ $\left..01, \eta^{2}=.06\right)$, mastery-approach $\left(F[1,694]=85.70, p<.01, \eta^{2}=.11\right)$, and mastery-avoidance $\left(F[1,694]=14.72, p<.01, \eta^{2}=.02\right)$, compared to the Korean athletes. In contrast, Korean athletes reported marginally higher scores in performance-approach $(F[1,694]=3.66, p=.06$, 
$\left.\eta^{2}=.01\right)$, and performance-avoidance $\left(F[1,694]=22.03, p<.01, \eta^{2}=.06\right)$, compared to the Singaporean athletes.

\section{MODERATION EFFECT OF PERCEIVED COMPETENCE}

Table 2 shows the means and standard deviations of the two groups in the key constructs for the higher and lower competency groups. The higher competency group $(M=6.20, S D=0.47)$ reported a significantly higher competency score, compared to the lower competency group $(M=$ $4.05, S D=0.58)$. Interestingly, the higher competency group also reported higher scores in all of the four achievement goals and the six subscales of the TDEQ (all $p$ s $<.05$, except for lack of quality preparation, $n s$ ). Chi-square tests revealed no significant differences among gender in the two groups, but there was a significant country effect. That is, there were significantly more Singaporean athletes in the higher competency group, compared to the Korean athletes.

In terms of the correlation tests, the higher competency group appeared to have a moderate correlation among the four achievement goals, while the lower competency group had much stronger associations among them, particularly between the two mastery goals (masteryapproach and mastery-avoidance) and two approach goals (mastery-approach and performanceapproach), and between performance-approach and mastery-avoidance goals. In the two competency groups, similar patterns emerged between the six subscales of the TDEQ. That is, long term development focus was positively correlated with communication, support network, and long term development fundamentals. Lack of quality preparation was positively correlated with lack of understanding the athletes; communication was positively related to support network and long term development fundamentals; and support network was also highly correlated with long term development fundamentals (see Table 3). 
In the path analyses, the moderation effects of perceived competence between the six talent development environment factors and four achievement goals was tested. The results are shown in Table 4. For the higher competency group, long term development focus positively predicted mastery-approach goal and performance goals. Long term development fundamentals positively predicted performance goals and mastery-avoidance, while communication was a negative predictor of mastery-approach goal. Support network positively predicted masteryavoidance goal but was negatively associated with performance-approach goal. Among the lower competency group, long term development focus positively predicted mastery goals and performance-approach goals. On the other hand, lack of quality preparation was a negative predictor of all four achievement goals. The results provided evidence that perceived competence moderated the relationship between the talent development environment and achievement goal adoption.

\section{DISCUSSION}

There is a recent call to examine the effects of gender and cultural contexts in which knowledge is developed and changed over time when studying competence and achievement motivation [16]. This study marks an exploratory attempt to examine the gender and country effects in the talent development environment and achievement goals, as well as the moderating role of perceived competence in the relationships between the two domains.

In answering the first research question, the results showed that there were significant differences among the male and female athletes in competence, achievement goals, and perceptions of the talent development environment. Male athletes showed higher competence, higher performance-approach goal, and lower mastery-avoidance goal, compared to the female 
athletes. The results are consistent with the previous studies in sport and physical education domains [8, 20]. Hyde and Durik [16] suggested that gender effects on achievement goals are domain specific. Sports may favour the male athletes over the female athletes due to the socialization process. Therefore, it is logical for male athletes to reported higher scores in performance-approach goal. However, there were no differences in mastery-approach and performance-avoidance goals between male and female athletes. This could be due to the talent development environment in which the athletes are trained in. Male athletes tended to view the talent development environment as having more long term developmental focus, communication, and long term development fundamentals than the female athletes. These factors may have contributed to the insignificant differences in mastery-approach and performance-avoidance goals between the two genders.

In terms of the differences between the two countries, Singapore athletes reported higher perceived competence, mastery goals, and lower performance-avoidance goal compared to the Korean athletes. It has been a common finding from motivation scholars that an emphasis on mastery goal increases the perception of competence and greater effort [23], where the relationship between perceived competence and effort is mediated by intrinsic motivation [24]. One possible explanation behind the results was that Singapore athletes were more task-oriented (e.g., learning of skill), and more likely enjoy the sports they were involved in. However, Korean athletes might feel more competitive and were more incline to outperform others for being selected in the national team or earning medals in the zero-sum nature of competitions. In terms of the talent development environment, there was not much difference between the athletes from the two nations, except that Singaporean athletes tended to report a lack of quality preparation and support network in their training environment. It can be argued that Korea has initiated the 
talent development programs earlier than Singapore and accumulated knowledge and skills in training from more extensive experiences from numerous internationally competitive sporting events. Korean coaches and administrators could provide athletes with better quality practices and training along with a wide range of support from the government bodies, the national sport science institute, senior athletes, and parents.

One of the major contributions of the current study was to examine the roles of perceived competence in the perception of the talent development environment and goal achievement adoption. There has been a strong emphasis on the role of perceived competence in determining achievement goals and forming patterns of achievement behaviors [16]. According to the achievement goal approach [11-13], competence is seen as the antecedent of achievement goals. However, very few studies have examined the effects that the varying levels of competence might have on achievement goal adoption and perceptions of the talent development environment. In addressing research question two, the results of the present study found that when the athletes were divided into higher and lower perceived competency groups, the patterns of goal adoption and perceptions of the environment appeared even clearer than the gender effect. Firstly, athletes who were higher in competence reported higher mastery goals and performance goals, regardless of whether it was approach or avoidance. Second, athletes who had higher perceived competence responded more positively toward the talent development environment. However, due to the cross-sectional design of the present study, we were not able to draw any causality relationships.

The third research question was concern with the moderating effects of perceived competence on the impacts of the talent development environmental factors on athletes' achievement goals. Regardless of the level of perceived competence, long term development 
focus predicted mastery-approach and performance-approach goals. When perceived competence was getting higher, long term development focus was not a significant predictor of masteryavoidance goal, however, when perceived competence was getting lower, long term development focus predicted mastery-avoidance goal. This shows that perceived competence moderates the relationships between the talent development environment and achievement goal adoption. Elliot (2005) suggests that both mastery-approach and performance approach goals contribute to positive effects. Thus, one important practical implication is that coaches are suggested to create a conducive talent development environment to promote athletes' approach goals. A special emphasis should be placed on long term development focus as this factor was shown to influence athletes' approach despite of their competence level. To this end, coaches should bear a longterm development vision in mind while planning their training program (e.g., allow athletes to make mistakes in training and educate athletes to take responsibility for their own development). In an effort to promote approach goals through manipulating other talent development environmental factors (e.g., communication and quality preparation), coaches should take athletes' competency level into consideration. For example, lack of communication will negatively affect mastery-approach goal adoption for those athletes with high competency level. This implies that effective athlete-coach communication (e.g., provide feedback and let athletes understand rationale of training) is important for facilitating the adoption of mastery-goal approach for those athlete with high competence.

\section{LIMITATION AND IMPLICATIONS}

The data for the current study were collected through one time-point therefore, causality cannot be inferred. Although the six subscales of the TDEQ showed adequate reliability, there is an indication that the psychometric properties of the TDEQ require further work. For example, the 
correlations between some of the subscales (long term development focus, communication, support network, and long term development fundamentals) are quite high [7,8]. Given the high attention of talent development paid by international audience $[1,2]$, there is a pressing need to re-examine the factorial structure of this inventory (e.g., using confirmatory factor analysis).

While the study utilized data collected from one of the most renowned elite sports schools each in Singapore and Korea, they are yet representative of all elite athletes enrolled in the talent development program in both countries. Thus, the samples from the secondary-level schools could limit the generalizability of the results. Future research may include more systematic replications of the current research design with samples from other populations (e.g., high school level and college level) to establish the external validity of the current results.

Lastly, the study considered possible effects of culture as a situational characteristic on the talent development environment and achievement goals. In addition, another significant situational characteristic, task dependence, should be considered as a possible inspiring factor in the domains of interest. In athletics, an individual sports is considered as a relatively low dependent task which requires less interaction with others, and a team sports is considered as relatively a high dependent task which requires high interactions with others in a team [25]. Athletes in team sports can perform their task competently to meet goals which are established differently from goals for athletes participating in individual sports. In addition, there might be different preferences toward the environmental factors between team and individual sports athletes. In a future study, it should be hypothesized that there is a task dependence difference in terms of the talent development environment and achievement goals. 


\section{References}

1. Abbott, A. and Collins, D., Eliminating the Dichotomy between Theory and Practice in Talent Identification and Development: Considering the Role of Psychology, Journal of Sports Sciences, 2004, 22(5), 395-408.

2. Baker, J. and Schorer, J., Identification and Development of Talent in Sport: Introduction to the Special Issue, Talent Development \& Excellence, 2010, 2(2), 119-121.

3. Martindale, R. J. J., Collins, D. and Abraham, A., Effective Talent Development: The Elite Coach Perspective within UK Sport, Journal of Applied Sports Psychology, 2007, 19(2), 187-206.

4. Abbott, A., Collins, D., Martindale, R. and Sowerby, K., Talent identification and development: An Academic Review, Sportscotland, Edinburgh, 2002.

5. Ericsson, K. A., Krampe, R. T. and Tesch-Römer, C., The Role of Deliberate Practice in the Acquisition of Expert Performance, Psychological Review, 1993, 100(3), 363-406.

6. Bloom, B. S., Developing Talent in Young People, Ballantine, New York, 1985.

7. Martindale, R. J. J., Collins, D., Wang, C. K. J., McNeill, M., Lee, K. S., Sproule, J. and Westbury, T., Development of the Talent Development Environment Questionnaire for Sport, Journal of Sports Sciences, 2010, 28(11), 1209-1221.

8. Wang, C. K. J., Sproule, J., McNeill, M., Martindale, R. J. J. and Lee, K. S., Impact of the Talent Development Environment on Achievement Goals and Life Aspirations in Singapore, Journal of Applied Sport Psychology, 2011, 23(3), 263-276.

9. Nicholls, J. G., The Competitive Ethos and Democratic Education, Harvard Universtiy Press, Cambridge, 1989. 
10. Pintrich, P. R., Multiple Goals, Multiple Pathways: The Role of Goal Orientation in Learning and Achievement, Journal of Educational Psychology, 2000, 92, 544-555.

11. Elliot, A. J., Approach and Avoidance Motivation and Achievement Goals, Educational Psychologist, 1999, 34(3), 169-189.

12. Elliot, A. J., A Conceptual History of the Achievement Goal Construct, in: Elliot, A. and Dweck, C., eds., Handbook of Competence and Motivation, Guilford Press, New York, $2005,52-72$.

13. Elliot, A. J. and McGregor, H. A., A $2 \times 2$ Achievement Goal Framework, Journal of Personality and Social Psychology, 2001, 80, 501-519.

14. McGregor, H. A. and Elliot, A. J., Achievement Goals as Predictors of AchievementRelevant Processes Prior to Task Engagement, Journal of Educational Psychology, 2002, 94, 381-395.

15. Middleton, M. and Midgley, C., Avoiding Demonstration of Lack of Ability: An Underexplored Aspect of Goal Theory, Journal of Educational Psychology, 1997, 89, 710-718.

16. Hyde, J. S. and Durik, A. M., Gender, Competence, and Motivation, in: Elliot, A. and Dweck, D., eds., Handbook of Competence and Motivation, Guilford Press, New York, $2005,375-391$.

17. Biddle, S. J. H., Wang, C. K. J., Kavussanu, M. and Spray, C. M., Correlates of Achievement Goal Orientations in Physical Activity: A Systematic Review of Research from 1990-2000, European Journal of Sport Sciences, 2003, 3, 1-20.

18. Hoffman, L. W., Early Childhood Experiences and Women's Achievement Motives, Journal of Social Issues, 1972, 28(2), 129-155. 
19. Brislin, R. W., Applied Cross-Cultural Psychology, Sage, Newbury Park, 1990.

20. Wang, C. K. J., Biddle, S. J. H. and Elliot, A. J., The $2 \times 2$ Achievement Goal Framework in a Physical Education Context, Psychology of Sport \& Exercise, 2007, 8(2), 147-168.

21. La Guardia, J. G., Ryan, R. M., Couchman, C. E. and Deci, E. L., Within-Person Variation in Security of Attachment: A Self-Determination Theory Perspective on Attachment, Need Fulfillment, and Well-Being, Journal of Personality and Social Psychology, 2000, 79(3), 367-384.

22. Bentler, P. M., EQS for Windows (Version 6.1), Multivariate Software, Encino, 2006.

23. Duda, J. L., Relationship between Taks and Ego Orientations and the Perceived Purpose of Sport among High School Athletes, Journal of Sport \& Exercise Psychology, 1989, 11, 318-335.

24. Deci, E. L. and Ryan, R. M., Intrinsic Motivation and Self-Dtermination in Human Behavior, Plenum Press, New York, 1985.

25. Chelladurai, P., Discrepancy between Preferences and Perceptions of Leadership Behavior and Satisfaction of Athletes in Varying Sports, Journal of Sport Psychology, $1984,6,27-41$. 